\title{
Antibiotic Prescribing in 4 Assisted-Living Communities: Incidence and Potential for Improvement
}

\author{
Philip D. Sloane, MD, MPH; ${ }^{1,2}$ Sheryl Zimmerman, PhD; ${ }^{2,3}$ David Reed, $\mathrm{PhD} ;{ }^{2}$ Anna Song Beeber, PhD, $\mathrm{RN} ;{ }^{4}$ \\ Latarsha Chisholm, $\mathrm{PhD} ;{ }^{2}$ Christine Kistler, MD, MASc; ${ }^{1}$ Christine Khandelwal, MD; $^{1}$ \\ David J. Weber, MD, MPH; ${ }^{5}$ C. Madeline Mitchell, MURP ${ }^{2}$
}

\begin{abstract}
овлестіле. To describe the prevalence, characteristics, and appropriateness of systemic antibiotic use in assisted living (AL) and to conduct a preliminary quality improvement intervention trial to reduce inappropriate prescribing.
\end{abstract}

DESIGN. Pre-post study, with a 13-month intervention period.

SETTING. Four AL communities.

PARTICIPANTs. All prescribers, all AL staff who communicate with prescribers, and all patients who had an infection during the baseline and intervention periods.

INTERVENTION. A standardized form for AL staff, an online education course and 5 practice briefs for prescribers, and monthly quality improvement meetings with AL staff.

MEASUREMENTs. Monthly inventory of all systemic antibiotic prescriptions; interviews with the prescriber, AL staff member, closest family member, and patient (when capable) regarding 85 antibiotic prescribing episodes (30 baseline, 55 intervention), with data review by an expert panel to determine prescribing appropriateness.

RESULTS. The mean number of systemic antibiotic prescriptions was 3.44 per 1,000 resident-days at baseline and 3.37 during the intervention, a nonsignificant change $(P=.30)$. Few prescribers participated in online training. AL staff use of the standardized form gradually increased during the program. The proportion of prescriptions rated as probably inappropriate was $26 \%$ at baseline and $15 \%$ during the intervention, a nonsignificant trend $(P=.25)$. Drug selection was largely appropriate during both time periods.

CONCLUSIONS. AL antibiotic prescribing rates appear to be approximately one-half those seen in nursing homes, with up to a quarter being potentially inappropriate. Interventions to improve prescribing must reach all physicians and staff and most likely will require long time periods to have the optimal effect.

Infect Control Hosp Epidemiol 2014;35(S3):S62-S68

Antimicrobial resistance among bacterial pathogens is an important and growing public health concern, ${ }^{1,2}$ and inappropriate overprescribing is believed to be a contributing factor. ${ }^{3}$ Since antibiotic prescribing rates are high in nursing homes (ranging from 3 to 5 prescriptions per resident annually), ${ }^{4-9}$ concern has been raised about potentially inappropriate prescribing in these settings. ${ }^{10} \mathrm{~A}$ few studies of attempts to reduce antibiotic overprescribing in nursing homes have been published, and these have met with mixed results. ${ }^{1-13}$

Due to changes in long-term care regulation and financing, assisted living (AL) has grown in recent decades to rival nursing homes in the number of chronically ill older persons served. ${ }^{14,15}$ Since nursing home and AL residents overlap con- siderably in terms of their medical problems and functional status, ${ }^{16}$ by inference it seems likely that antibiotic overprescribing may also be a problem in AL. However, in spite of the large and growing population served by $\mathrm{AL}$, this setting has received virtually no attention in attempts to either describe or optimize antibiotic prescribing. ${ }^{8}$ Studying this setting would be important both because of the number of persons served and because its organizational structure differs considerably from that of nursing homes, making extrapolation of results from nursing home studies not necessarily appropriate to AL.

To begin to understand the prevalence and characteristics of antibiotic use in AL communities and to explore the po-

Affiliations: 1. Department of Family Medicine, University of North Carolina at Chapel Hill, North Carolina; 2. Cecil G. Sheps Center for Health Services Research, University of North Carolina at Chapel Hill, North Carolina; 3. School of Social Work, University of North Carolina at Chapel Hill, North Carolina; 4. School of Nursing, University of North Carolina at Chapel Hill, North Carolina; 5. Department of Medicine, University of North Carolina at Chapel Hill, North Carolina.

Received April 7, 2014; accepted April 13, 2014; electronically published September 15, 2014

(C) 2014 by The Society for Healthcare Epidemiology of America. All rights reserved. 0899-823X/2014/35S3-0010\$15.00. DOI: 10.1086/677821 
tential to reduce inappropriate antibiotic prescribing in this setting, we designed, implemented, and evaluated in $4 \mathrm{AL}$ communities a yearlong quality improvement program aimed at optimizing antibiotic prescribing. Our multicomponent intervention included physician training, introduction of a standardized acute problem recording and communication form, and a series of staff training and quality improvement activities within each participating AL community. This article describes the intervention and presents antibiotic prescribing at baseline and during the intervention.

\section{METHODS}

\section{Design and Sample}

Four AL communities participated in the study. Selection criteria included location within 30 miles of the project office, census of at least 40 residents, willingness of the administrator and staff to participate in the quality improvement program and the associated evaluation, and an auditable system of recording medication administration. In addition, because one medical practice was extensively involved in AL care in the region, communities were chosen that varied in the proportion of residents managed by that practice.

\section{Assessing Prescribing Rates}

To assess antibiotic prescription rates in each AL community, a nurse data collector reviewed all medication records during the baseline period (August-October 2010 [depending on site] through February 2011) and the intervention period (March 2011 through March 2012). For each systemic (ie, not topically applied) antibiotic prescription, the medical record was reviewed to obtain the following information: the resident receiving the medication, prescriber, drug name, start and stop dates, administration route, whether the prescription was initiated within 2 days of an overnight hospital stay or emergency department visit, type of infection (urinary, respiratory, skin, gastrointestinal, preventive, or other), and whether the resident had advance directives related to antibiotic use.

\section{Intervention}

The intervention consisted of 4 main components: education of medical care providers/prescribers, introduction of a standardized 1-page medical care referral form (MCRF, available online) for $\mathrm{AL}$ staff to record and communicate signs and symptoms, a series of staff training and quality improvement activities within each AL community, and family/patient education. Each is described briefly below; additional details of the intervention have been published elsewhere. ${ }^{17}$

Provider/prescriber education. A 5-module Internet-based training program was developed and offered to all physicians, nurse practitioners, and physician assistants who had patients in the participating $\mathrm{AL}$ communities or had prescribed an antibiotic to a resident of a participating AL community during baseline data collection. The modules presented baseline an- tibiotic prescribing data for the participating AL communities and offered evidence-based training on antibiotic resistance and infection control in long-term care. Continuing education credit was offered by the School of Medicine of the University of North Carolina at Chapel Hill, and the 5 medical care providers in each $\mathrm{AL}$ community who had prescribed the most antibiotics at baseline were offered $\$ 250$ if they completed the 5 training modules. In addition, all active providers in each AL community ( $N=232-243$, depending on month) were mailed 1-page practice briefs summarizing guidelines on infection treatment and control every other month.

MCRF. On the basis of the literature, feedback from an expert panel, and interviews with staff of the participating AL communities, we developed a standardized form to be used by $\mathrm{AL}$ staff to record and communicate information about acute medical problems. AL staff were asked to use the form when a new medical problem occurred and communication with a provider was needed, indicating the presumed infection type, if applicable (urinary, skin/soft tissue, respiratory, or gastrointestinal), and completing a checklist of site-specific signs, symptoms, and, if available, laboratory results. In addition, the form included a blank section for a written description of the current problem, a space for vital signs, and a brief medical history checklist. The bottom of the form included space for providers to record orders, with the intent that forms would be faxed or otherwise returned after a visit to an emergency department, hospital, or physician office.

To determine the degree to which the MCRF was used and completed, a research assistant obtained copies of completed forms from each site, deleted personal identifiers, and entered data into the project database.

Staff training and quality improvement. Staff training consisted of an information session, in-service training, and nursespecific training. The information session was a 1 -time 30 minute session for all AL community staff; topics included the risks of antibiotic overuse, the problem with resistant organisms, and a general description of the quality improvement program. The in-service was a 45 -minute session for medication technicians, healthcare supervisors, managers, and licensed nurses; it focused on completion of the MCRF and included case studies demonstrating its use. Additional training for nurses (RNs, LPNs, and LVNs) focused on signs and symptoms of infection and nursing-specific interventions to provide symptom management when an antibiotic is not indicated.

For the quality improvement component of the program, each AL community identified a quality improvement team that met monthly with the project team to review the previous month's data on infection incidence and MCRF use, discuss facilitators and barriers of MCRF use (including strategies to increase use), and discuss communication with physicians related to acute medical problems and use of MCRF data. In addition, the data collector who gathered the MCRFs weekly provided encouragement for their use.

Family and patient education. Each AL community was asked to distribute to all residents and their families an in- 
formational brochure that described the quality improvement program and highlighted the risks of antibiotic overuse. At the request of 2 communities, project team members presented information about the program at a family night.

\section{Case Studies to Assess Appropriateness of Antibiotic Prescriptions}

To determine the appropriateness of antibiotic prescribing, case studies of a stratified sample of antibiotic prescriptions were conducted at 3 time periods: once prior to the initiation of the intervention and twice during the intervention period. Up to 36 cases were included for each time period. Cases were excluded if the antibiotic was for prevention (eg, before dental procedures), had occurred within 2 days of a hospitalization, or involved a resident previously selected as a case. Stratification of the sample was by AL community and type of infection (urinary, respiratory, skin, or gastrointestinal/ other). After a case was identified, consent was obtained from the resident or a family member (if the resident was unable) and interviews were conducted with (a) the resident (if able), (b) the family member most involved in the resident's care, (c) the staff member who contacted the prescriber or knew the resident best, and $(d)$ the prescriber. All respondents were asked a standardized series of questions regarding signs and symptoms, the decision-making process, and their opinions about how ill the person was and the likelihood of getting better without an antibiotic. The staff, resident, and family interviews were conducted by a nurse data collector; the prescriber interviews were conducted by a physician.

An expert panel consisting of a geriatrician, infectious disease specialist, and clinical pharmacist reviewed each case study and rated the appropriateness of prescribing the antibiotic on a 5 -point scale: $0=$ not appropriate, $1=$ probably not appropriate, $2=$ uncertain, $3=$ probably appropriate, and $4=$ appropriate. Decisions were made by consensus. For interpretation purposes, a dichotomous "probably not appropriate" variable was created, consisting of all prescriptions judged by the panel to be not appropriate or probably not appropriate. In addition, the expert panel judged the appropriateness of the antibiotic selection, given the diagnosis and clinical data provided (including culture results if available at the time of the prescription).

\section{Data Analysis}

Tests of change over time in the number of prescriptions per resident-day were conducted as generalized linear models using a negative binomial model to accommodate overdispersion and an offset for the number of resident-days. Response variables were the number of prescriptions generated within an AL community during a month for specific types of infections and for infections overall. Predictor variables were the intervention period (pre-post), community, and the interaction of intervention period and community. A ratio of the number of MCRFs completed to the number of prescriptions generated was calculated, and the association of this variable with the time elapsed since the intervention period began was tested with a linear regression model. Analyses were carried out using the GENLIN and REGRESSION commands in SPSS (ver. 18).

\section{RESULTS}

\section{Participating AL Communities}

The participating AL communities had a mean bed size of 94.5 (standard deviation [SD], 36.6). Three were for profit; 2 were part of a chain. Two had a full-time licensed nurse on staff (1 registered nurse, 1 licensed practical nurse). The mean number of primary care physicians managing patients in a study community was 33.0 (SD, 28.0). All 4 communities had at least 1 medical provider who made regular on-site visits (1 had 2 providers who did this), and the percentage of residents seen by providers who visited regularly averaged $41.5 \%$ (SD, 19.4\%).

\section{Preintervention Incidence and Patterns of Antibiotic Prescriptions}

During the preintervention baseline, the rate of new prescriptions of systemic antibiotics in the $4 \mathrm{AL}$ communities ranged from 2.53 to 4.56 prescriptions per 1,000 patient-days, with a mean of 3.44 (Table 1). The most common reason for prescriptions was urinary tract disease (mean rate, 1.67 prescriptions per 1,000 resident-days), followed by respiratory disease ( 1.00 prescriptions per 1,000 resident-days).

All prescriptions were for oral medications. The most commonly prescribed drugs (Table 2) for presumed urinary tract infections were ciprofloxacin ( $36 \%$ of prescriptions), nitrofurantoin (14\%), trimethoprim-sulfamethoxazole (13\%), levofloxacin (10\%), and cephalexin (8\%). The most commonly prescribed drugs for respiratory infections were levofloxacin (28\%), moxifloxacin $(20 \%)$, azithromycin (18\%), ciprofloxacin $(10 \%)$, and amoxicillin clavulanate $(10 \%)$. The timing of the prescriptions was such that $22 \%$ were initiated within 2 days of an overnight hospitalization and an additional $12 \%$ within 2 days of an emergency department visit (Table 2).

\section{Participation of AL Staff and Prescribers in Intervention Activities}

The information session for staff was conducted in all $4 \mathrm{AL}$ communities and was attended by a total of 59 staff. Separate in-service sessions that focused on the use of the MCRF were attended by 37 nurses, medication technicians, or care aides across the 4 communities. Two communities requested an additional in-service session because of staff turnover during the intervention period; 17 staff attended across the $2 \mathrm{com}$ munities. In addition, 47 monthly quality improvement meetings were held across the 4 communities between March 2011 and March 2012, with the number of staff in attendance ranging from 1 to 5 . These meetings occurred monthly at 3 sites; at a fourth site only 7 occurred because of the absence of a site coordinator for several months. 
TABLE 1. Incidence of Antibiotic Prescribing in 4 Assisted-Living Communities by Number of Prescriptions and Rate of Prescribing

\begin{tabular}{|c|c|c|c|c|c|}
\hline & \multicolumn{2}{|c|}{$\begin{array}{c}\text { Preintervention } \\
\text { (August 2010-February 2011) }\end{array}$} & \multicolumn{2}{|c|}{$\begin{array}{c}\text { During intervention } \\
\text { (March 2011-March 2012) }\end{array}$} & \multirow[b]{2}{*}{$\begin{array}{c}P \text { value for } \\
\text { difference in rates }\end{array}$} \\
\hline & $\begin{array}{c}\text { No. of } \\
\text { prescriptions }\end{array}$ & $\begin{array}{l}\text { Rate, cases per } \\
1,000 \text { patient-days }\end{array}$ & $\begin{array}{c}\text { No. of } \\
\text { prescriptions }\end{array}$ & $\begin{array}{l}\text { Rate, cases per } \\
1,000 \text { patient-days }\end{array}$ & \\
\hline \multicolumn{6}{|l|}{ All infections } \\
\hline Community A & 47 & 3.79 & 150 & 3.48 & $\ldots$ \\
\hline Community B & 61 & 3.75 & 176 & 3.46 & $\ldots$ \\
\hline Community $\mathrm{C}$ & 43 & 4.56 & 114 & 3.60 & $\ldots$ \\
\hline Community D & 55 & 2.53 & 223 & 3.16 & $\ldots$ \\
\hline All communities & 206 & 3.44 & 663 & 3.37 & .30 \\
\hline \multicolumn{6}{|c|}{ Urinary tract infections only } \\
\hline Community A & 28 & 2.26 & 68 & 1.35 & $.031^{\mathrm{b}}$ \\
\hline Community B & 26 & 1.60 & 104 & 2.35 & .08 \\
\hline Community $\mathrm{C}$ & 23 & 2.44 & 61 & 1.93 & .34 \\
\hline Community D & 23 & 1.06 & 77 & 1.02 & .89 \\
\hline All communities & 100 & 1.67 & 310 & 1.55 & .64 \\
\hline \multicolumn{6}{|c|}{ Respiratory infections only } \\
\hline Community A & 9 & 0.73 & 36 & 0.91 & .59 \\
\hline Community B & 21 & 1.29 & 32 & 0.33 & $<.001^{\circ}$ \\
\hline Community $\mathrm{C}$ & 13 & 1.38 & 22 & 0.46 & .036 \\
\hline Community D & 17 & 0.78 & 70 & 1.00 & .12 \\
\hline All communities & 60 & 1.00 & 160 & 0.74 & .39 \\
\hline \multicolumn{6}{|l|}{ Skin infections only } \\
\hline Community A & 2 & 0.16 & 16 & 0.47 & $\ldots$ \\
\hline Community B & 5 & 0.31 & 24 & 0.57 & $\cdots$ \\
\hline Community $\mathrm{C}$ & 2 & 0.21 & 16 & 0.71 & $\ldots$ \\
\hline Community D & 5 & 0.23 & 35 & 0.57 & $\ldots$ \\
\hline All communities & 14 & 0.23 & 91 & 0.57 & .004 \\
\hline
\end{tabular}

NOTE. Data were collected monthly from medication administration records.

a Differences in rates between preintervention and intervention periods were tested using a negative binomial regression model with an offset (adjustment) for the number of resident-days. $P$ values for individual communities are presented only where the interaction of intervention period (pre-post) and community was statistically significant.

${ }^{b}$ For the intervention by community interaction, $P=.042$.

c For the intervention by community interaction, $P<.001$.

The Internet-based educational program series for physicians, physician assistants, and nurse practitioners (5 modules, approximately 20 minutes each) was begun by 20 providers and completed by 16 .

Use of the MCRF gradually increased over the 13-month intervention period, from 12 forms during the first month to a peak of 68 near the end of the intervention period (Figure 1). During the early intervention months, far more prescriptions were written than MCRFs completed; by the end of the study, the number of forms slightly exceeded the number of prescriptions (but not all forms related to these cases).

\section{Comparison of Baseline Prescribing with Prescribing during the Intervention}

The overall rate of antibiotic prescribing during the intervention period was 3.37 prescriptions per 1,000 resident-days; it did not differ statistically $(P=.30)$ from the preintervention rate (Table 1). Rates of antibiotic prescribing did not change for either urinary or respiratory infections, and the prescribing rate for skin infections increased significantly (from 0.23 to 0.57 prescriptions per 1,000 resident-days; $P=.004$ ).
Results of expert panel rating of 30 baseline and 55 intervention case reports are displayed at the end of Table 2 . The proportion of prescriptions judged to be probably inappropriate was $26 \%$ at baseline and $15 \%$ during the intervention, a nonsignificant reduction $(P=.25)$. Drug selection was largely appropriate during both time periods: $90 \%$ at baseline and $85 \%$ during the intervention $(P=.74)$.

\section{DISCUSSION}

To our knowledge, this is the first report of infection rates and antibiotic use in AL communities, a contention that is supported both by our literature search and by a recent US Department of Health and Human Services position paper. ${ }^{10}$ Over a year and a half of observation, both at baseline and during a 13-month intervention period, we found that antibiotics are relatively common in AL settings and are most commonly prescribed for urinary and respiratory symptoms, as is true in nursing homes. ${ }^{18} \mathrm{~A}$ multicomponent intervention was used to reduce potentially inappropriate prescribing, and, although intervention uptake was limited and overall pre- 


\section{Project phase}

Preintervention Post-QI intervention

Route

Parenteral

Timing of prescription

Within 3 days of prior antibiotic use

Within 2 days of overnight hospitalization

Within 2 days of emergency department visit (without hospitalization)

Indication

Urinary tract infection

Respiratory infection

Skin infection

Gastrointestinal tract infection

Preventive

Other

Not noted

Advance directives for no antibiotics

Advance directive for no antibiotics on medical record

Most common antibiotics prescribed (all prescriptions), preintervention/QI intervention

1. Ciprofloxacin/1. Ciprofloxacin

2. Levofloxacin/2. TMP-SMZ

3. TMP-SMZ/3. Cephalexin

4. Cephalexin/4. Nitrofurantoin

5. Nitrofurantoin/5. Amoxicillin clavulanate

5. Azithromycin/6. Levofloxacin

Most common antibiotics prescribed for urinary infections, preintervention/QI intervention

1. Ciprofloxacin/1. Ciprofloxacin

2. Nitrofurantoin/2. Nitrofurantoin

2. TMP-SMZ/3. TMP-SMZ

4. Levofloxacin/4. Cephalexin

5. Cephalexin/5. Levoflaxacin

6. Amoxicillin clavulanate/6. Amoxicillin clavulanate

Most common antibiotics prescribed for respiratory infections, preintervention/QI intervention

1. Levofloxacin/1. Azithromycin

2. Moxifloxacin/2. Levofloxacin

3. Azithromycin/3. Moxifloxacin

4. Ciprofloxacin/4. Amoxicillin clavulanate

4. Amoxicillin clavulanate/5. Clarithromycin

Most common antibiotics prescribed for skin infections, preintervention/QI intervention

1. Cephalexin/1. Cephalexin

2. Amoxicillin clavulanate/2. TMP-SMZ

3. TMP-SMZ/3. Amoxicillin clavulanate

3. Ciprofloxacin/4. Ciprofloxacin

3. Levofloxacin/5 Clindamycin

6. Doxycycline/5. Doxycycline

Appropriateness of decision to prescribe $e^{a, b}$

$0=$ not at all

$1=$ a little

2 = somewhat

$3=$ mostly

$4=$ definitely

Mean appropriateness (0-4)

Was antibiotic selection appropriate? ${ }^{\text {a,c }}$

Yes

No
$206(100)$
$0(0)$

$24(12)$

46 (22)

25 (12)

$100(49)$

$60(29)$

$14(7)$

$2(1)$

$6(3)$

11 (5)

$13(6)$

$0(0)$

51 (25)

29 (14)

$19(9)$

$16(8)$

$15(7)$

$15(7)$

36 (36)

15 (14)

15 (13)

$10(10)$

8 (8)

3 (3)

17 (28)

$12(20)$

11 (18)

$6(10)$

$6(10)$

4 (29)

3 (21)

2 (14)

2 (14)

2 (14)

1 (7)

$n=30$

1 (3)

7 (23)

1 (3)

3 (10)

$18(60)$

3.0

$n=30$

27 (90)

3 (10)
457 (100)

0 (0)

29 (6)

59 (13)

40 (9)

210 (47)

$100(22)$

77 (17)

5 (1)

17 (4)

$12(3)$

$30(7)$

$0(0)$

$91(20)$

57 (12)

56 (12)

51 (11)

40 (9)

39 (9)

$64(31)$

46 (22)

33 (16)

24 (11)

14 (7)

11 (5)

$29(29)$

16 (16)

15 (15)

11 (11)

9 (9)

$20(26)$

$14(18)$

$10(13)$

8 (10)

7 (9)

7 (9)

$n=55$

2 (4)

6 (11)

$14(26)$

$19(35)$

14 (26)

2.7

$n=55$

47 (86)

8 (15)

Note. Data are no. (\%), unless otherwise indicated. All new prescriptions for systemic antibiotics are included regardless of site of prescription (hospital, emergency department, office, community). Baseline data collection occurred between August 1, 2010, and February 29, 2012 (length of data collection varied by assisted-living community); intervention data collection occurred between March 1, 2011, and March 31, 2012. Data were collected monthly from medication administration records. QI, quality improvement; TMP-SMZ, trimethoprim-sulfamethoxazole.

"Source of data was interviews conducted with prescribers, residents, and staff for a sample of antibiotic prescriptions.

b For the test of difference (preintervention vs intervention) in the proportion of prescriptions judged to be probably inappropriate (scores of 0 or 1$), P=.25$.

"For the exact test of difference between preintervention and intervention, $P=.74$. 


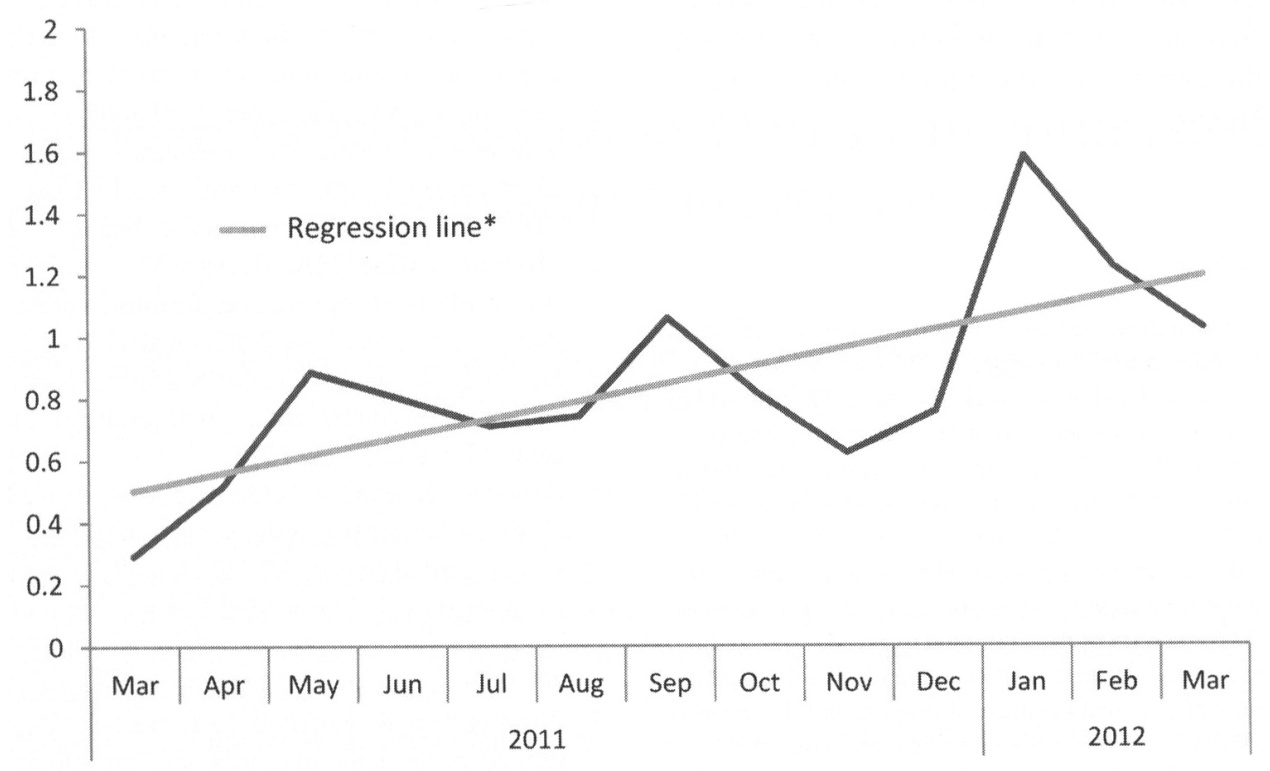

FIGURE 1. Ratio of the number of medical care referral forms completed to the number of prescriptions by intervention month. A gradual increase in this ratio indicates that, during the yearlong intervention period, the assisted-living staff slowly increased their adherence to this key aspect of the intervention. A linear regression with month of the intervention as predictor of the ratio was statistically significant $(P=.009)$.

scribing rates did not change, an encouraging although statistically nonsignificant trend toward improvement in prescribing appropriateness occurred (from $73 \%$ to $87 \%$ ). Of note are the data indicating that adherence to the project's main intervention, the MCRF, gradually increased over the yearlong intervention, suggesting that a quality improvement strategy that was carried out over a long period of time would be needed to change practice. This, combined with the limited power of this small sample, suggests that a larger, longer trial may indeed demonstrate significant change.

Given the consistency of our prevalence data across a year and a half in both the baseline and the intervention period, we feel reasonably confident that, if our settings are representative of $\mathrm{AL}$, the antibiotic prescription rate is between 3 and 4 prescriptions per 1,000 resident-days. Extrapolating from the work of Mylotte and Keagle, ${ }^{7}$ this would correspond to an infection rate of between 2 and 3 infections per 1,000 residentdays, with the remainder of prescriptions representing inappropriate prescribing for such conditions as asymptomatic bacteriuria, ${ }^{19}$ bacterial colonization of skin wounds, ${ }^{20}$ and viral or allergic respiratory conditions. ${ }^{21,22}$ Comparable figures for nursing homes vary widely, with the average infection rate around 4.5-5.0. ${ }^{723-25}$ Thus, on the basis of these limited data, we estimate that infection rates in $\mathrm{AL}$ are approximately one-half what they are in nursing homes. Data using consistent methods and more settings across both $\mathrm{AL}$ and nursing homes would be needed to develop more precise estimates for comparison. Furthermore, the variation noted among the $4 \mathrm{AL}$ communities (Table 1) is considerable, arguing for the need for a large sample size in a more definitive study to account for the anticipated large variance across sites.
Our expert panel identified $26 \%$ of baseline antibiotic prescriptions to be probably inappropriate. This is at the lower end of the range identified by nursing home studies (25\%$75 \%) .{ }^{8}$ However, appropriateness is subjective and many minimum standards for prescribing antibiotics, such as those of Loeb et $\mathrm{al}^{26}{ }^{26}$ are almost certainly too stringent. ${ }^{27}$ Our data suggesting a trend toward prescribing reduction (Table 1) are encouraging, but a larger study (ie, powered to detect clinically meaningful changes) with an intervention that has more penetrance (especially among physicians) would be needed to identify whether and to what extent antibiotic prescribing can indeed be changed in $\mathrm{AL}$.

Nursing home settings have been described as complex adaptive systems, as they operate in environments where several agents (ie, nursing home staff, administrators, doctors, and families) interact to provide care. ${ }^{27}$ Similar complex interactions also occur in $\mathrm{AL}$, where input from diverse sources may be beneficial for resident care but can also complicate the care process. ${ }^{28}$ For example, while AL community recruitment was not difficult (ie, administrative leadership agreed that infection management was an important issue, and we encountered no refusals during recruitment), a notable challenge related to educating medical providers, as is evidenced by the fact that only 20 of more than 250 persons approached to participate in online education actually accessed the site. With AL staff, we obtained better participation, but several other factors limited intervention effectiveness. These included difficulty changing staff behavior, which required frequent reminders of the form's availability, and high staff turnover, with a consequent need for retraining. In addition, program uptake was hampered by pervasive beliefs 
among staff that patients with a new or worsening cough need antibiotics, that urinary tract infection is a common cause of nonspecific symptoms, and that all positive urine cultures need treatment.

\section{ACKNOWLEDGMENTS}

Financial support. This research was conducted by the University of North Carolina at Chapel Hill under contract (290200710014I) to the Agency for Healthcare Research and Quality (AHRQ), Rockville, MD. The authors of this article are responsible for its content. No statement may be construed as the official position of AHRQ, US Department of Health and Human Services. P.D.S. is a recipient of Pioneer Award PIO-04-1004 from the US Alzheimer's Association.

Potential conflicts of interest. D.J.W. reports receiving grants from AHRQ during the conduct of the study and personal fees from Pfizer, Merck, Germitec, Johnson \& Johnson, and Clorox outside the submitted work. All other authors report no conflicts of interest relevant to this article. All authors submitted the ICMJE Form for Disclosure of Potential Conflicts of Interest, and the conflicts that the editors consider relevant to this article are disclosed here.

Address correspondence to Philip D. Sloane, MD, MPH, William B. Aycock Family Medicine Building, Campus Box 7595, Chapel Hill, NC 27599 (philip_sloane@med.unc.edu).

\section{REFERENCES}

1. Neu HC. The crisis in antibiotic resistance. Science 1992; 257(5073):1064-1073.

2. Croft AC, D'Antoni AV, Terzulli SL. Update on the antibacterial resistance crisis. Med Sci Monit 2007;13(6):RA103-RA118.

3. Gonzales R, Steiner JF, Lum A, et al. Decreasing antibiotic use in ambulatory practice: impact of a multidimensional intervention on the treatment of uncomplicated acute bronchitis in adults. JAMA 1999;281(16):1512-1519.

4. Warren JW, Palumbo FB, Fitterman L, et al. Incidence and characteristics of antibiotic use in aged nursing home patients. J Am Geriatr Soc 1991;39(10):963-972.

5. Katz PR, Beam TR Jr, Brand F, et al. Antibiotic use in the nursing home: physician practice patterns. Arch Intern Med 1990;150(7): 1465-1468.

6. Benoit SR, Nsa W, Richards CL, et al. Factors associated with antimicrobial use in nursing homes: a multilevel model. $J$ Am Geriatr Soc 2008;56(11):2039-2044.

7. Mylotte JM, Keagle J. Benchmarks for antibiotic use and cost in long-term care. J Am Geriatr Soc 2005;53(7):1117-1122.

8. Nicolle LE, Bentley DW, Garibaldi R, et al; SHEA Long-TermCare Committee. Antimicrobial use in long-term-care facilities. Infect Control Hosp Epidemiol 2000;21(8):537-545.

9. Zimmer JG, Bentley DW, Valenti WM, et al. Systemic antibiotic use in nursing homes: a quality assessment. J Am Geriatr Soc 1986;34(10):703-710.

10. US Department of Health and Human Services. National Action Plan to Prevent Health Care-Associated Infections: Road Map to Elimination. Chapter 10, "Long-Term Care Facilities." http:// www.hhs.gov/ash/initiatives/hai/actionplan/ltc_facilities508.pdf. Published 2013. Accessed December 21, 2013.
11. Loeb M, Brazil K, Lohfeld L, et al. Effect of a multifaceted intervention on number of antimicrobial prescriptions for suspected urinary tract infections in residents of nursing homes: cluster randomised controlled trial. BMJ 2005;331(7518):669A-672A.

12. Schwartz DN, Abiad H, DeMarais PL, et al. An educational intervention to improve antimicrobial use in a hospital-based LTC facility. J Am Geriatr Soc 2007;55(8):1236-1242.

13. Monette J, Miller MA, Monette M, et al. Effect of an educational intervention on optimizing antibiotic prescribing in LTC facilities. J Am Geriatr Soc 2007;55(8):1231-1235.

14. National Center for Health Statistics. Fast Stats A to Z. http:// www.cdc.gov/nchs/fastats/nursingh.htm. Published 2013. Accessed December 21, 2013.

15. Park-Lee E, Caffrey C, Sengupta M, et al. Residential care facilities: a key sector in the spectrum of long-term care providers in the United States. NCHS Data Brief 2011;(78):1-8.

16. Zimmerman S, Gruber-Baldini AL, Sloane PD, et al. Assisted living and nursing homes: apples and oranges? Gerontologist 2003:43(suppl 2):107-117.

17. Zimmerman S, Mitchell CM, Beeber AS, et al. Strategies to reduce potentially inappropriate antibiotic prescribing in assisted living and nursing homes. Adv Prev Control Healthc Assoc Infect (forthcoming).

18. Tsan L, Langberg R, Davis C, et al. Nursing home-associated infections in Department of Veterans Affairs community living centers. Am J Infect Control 2010;38(6):461-466.

19. Nicolle LE. Asymptomatic bacteriuria: when to screen and when to treat. Infect Dis Clin North Am 2003;17(2):367-394.

20. Sibbald RG, Woo K, Ayello EA. Increased bacterial burden and infection: the story of NERDS and STONES. Adv Skin Wound Care 2006;19(8):447-461.

21. Arroll B, Kenealy T, Falloon K. Are antibiotics indicated as an initial treatment for patients with acute upper respiratory tract infections? a review. $N Z$ Med J 2008;121(1284):64-70.

22. Smucny JJ, Becker LA, Glazier RH, et al. Are antibiotics effective treatment for acute bronchitis? a meta-analysis. J Fam Pract 1998;47(6):453-460.

23. Pennsylvania Patient Safety Authority. 2009 Annual Report. http://patientsafetyauthority.org/Documents/Annual_Report _2009.pdf. Published 2010. Accessed December 21, 2013.

24. Stevenson KB, Moore J, Colwell H, et al. Standardized infection surveillance in long-term care: interfacility comparisons from a regional cohort of facilities. Infect Control Hosp Epidemiol 2005; 26(3):231-238.

25. Koch AM, Eriksen HM, Elstrøm P, et al. Severe consequences of healthcare-associated infections among residents of nursing homes: a cohort study. J Hosp Infect 2009;71(3):269-274.

26. Loeb M, Bentley DW, Bradley S, et al. Development of minimum criteria for the initiation of antibiotics in residents of long-termcare facilities: results of a consensus conference. Infect Control Hosp Epidemiol 2001;22(2):120-124.

27. Juthani-Mehta $M$, Tinetti $M$, Perrelli E, et al. Diagnostic accuracy of criteria for urinary tract infection in a cohort of nursing home residents. J Am Geriatr Soc 2007;55(7):1072-1077.

28. Anderson RA, Issel LM, McDaniel RR Jr. Nursing homes as complex adaptive systems: relationship between management practice and resident outcomes. Nurs Res 2003;52(1):12-21. 